\title{
An Update on the Use of Exhaled Breath Analysis for the Early Detection of Lung Cancer
}

\author{
Nir Peled' \\ Vered Fuchs ${ }^{2}$ \\ Emily $\mathrm{H}$ Kestenbaum ${ }^{2}$ \\ Elron Oscar ${ }^{2}$ \\ Raul Bitran ${ }^{3}$
}

'Shaare Zedek Medical Center, The Hebrew University, Jerusalem, Israel; ${ }^{2}$ Faculty of Health Sciences, Ben-Gurion University of the Negev, Beer-Sheva, Israel; ${ }^{3}$ The Legacy Heritage Oncology Center \& Dr. Larry Norton Institute, Soroka Medical Center, Beer-Sheva, Israel
Correspondence: Nir Peled

Shaare Zedek Medical Center, 12, Shmuel

Beit St, Jerusalem, 9103102, Israel

Tel +972587040620

Email nirp@szmc.org.il

\begin{abstract}
Lung cancer has historically been the main responsible for cancer associated deaths. Owing to this is our current inability to screen for and diagnose early pathological findings, preventing us from a timely intervention when cure is still achievable. Over the last decade, together with the extraordinary progress in therapeutical alternatives in the field, there has been an ongoing search for a biomarker that would allow for this. Numerous technologies have been developed but their clinical application is yet to come. In this review, we provide an update on volatile organic compounds, a non-invasive method that can hold the key for detecting early metabolic pathway changes in carcinogenesis. For its compilation, web-based search engines of scientific literature such as PubMed were explored and reviewed, using articles, research, and papers deemed meaningful by authors discretion. After a brief description, we depict how this technique can complement current methods and present the value of electronic noses in the identification of the "breathprint". Lastly, we bring some of the latest updates in the field together with the current limitations and final remarks.
\end{abstract}

Keywords: lung cancer, volatile organic compounds, VOC, electronic nose, E-nose, GC-MS, biomarkers

\section{Intro: The Need for New Screening Methods}

Cancer continues to be the second deadliest disease worldwide. ${ }^{1,2}$ Amongst it, lung cancer claims the most lives, making up one in every four. While its incidence is not expected to raise, it will remain as the deadliest, accounting for more deaths than the next three on the list combined (prostate, breast, and colon). ${ }^{3}$

Responsible for this epidemiological finding, together with the high rates of smoking across all population groups, ${ }^{4}$ is the fact that we still do not count with proper screening methods which allow for early disease detection.

As brought down in the recently published NCCN 2021 Lung Cancer Screening Guidelines, ${ }^{5}$ low-dose computed tomography (LDCT) remains the only recommended and clinically approved screening tool. And while it has been implemented in different programs across the map, there is still a disputed claim about its efficacy in reducing the mortality rate, particularly because accurate criteria to define high risk groups has not reached consensus. ${ }^{6,7}$ Moreover, what makes it such an unattractive means is its high cost, overdiagnosis, and impracticality in everyday use. Literature reports up to $94 \%$ false positives, ${ }^{8}$ which lead to unnecessary interventions, ${ }^{9}$ high burden on the healthcare systems, and patient anxiety. ${ }^{10}$ 
In contraposition, mammography for example, has reduced breast cancer mortality in $20 \%$ according to the WHO. ${ }^{11}$ As for Colorectal cancer, numbers are even more impressive, with studies reporting up to a $61 \%$ decrease in mortality and $89 \%$ reduction in incidence with the introduction of colonoscopies. ${ }^{12,13}$ The case is similar with prostate cancer ${ }^{14}$ and PSA screening.

It is clear, thus, that what holds the key for lowering mortality rates and disease burden is discovering better screening tools that will allow for early diagnosis, while surgical resection can still provide a cure for the disease. ${ }^{15}$

Fortunately, together with the exponential advance in the treatment alternatives that have become available in the last decade, research for an early screening method has not stayed behind. While there are still no clinically validated methods, many platforms are being explored with promising results and even active clinical trials. ${ }^{16}$ Between others, worthy of notice are liquid biopsies, ${ }^{17}$ miRNA,${ }^{18}$ DNA methylation, ${ }^{19}$ nasal epithelial cells, ${ }^{20}$ and autoantibodies. ${ }^{21}$

In the current review, we provide an update on Volatile Organic Compounds (VOC), with emphasis on the latest studies and a short description of Exhaled Breath Condensate (EBC), the non-volatile component of exhaled breath.

\section{VOCs: What and Why}

VOCs are small molecular mass compounds that have a high vapor pressure and a low boiling point. ${ }^{22}$ This, in turn, allows for their detection and measurement in exhaled breath. They are produced by every living organism ${ }^{23}$ and, as explained in our previous review, ${ }^{24}$ they are believed to reflect metabolic processes at the tissue level such as inflammation and oxidative stress. They can be measured not only on exhaled air, but on a wide range of human matrices such as blood, urine, feces, pleural effusions, and cell lines among others. ${ }^{25}$

The sum of the VOCs has been coined as the "volatome," 26 or "breathome" when referring to its exhaled part. 22,26

One of the most recent discoveries in the field of origins and nature of VOCs is the finding that they serve as cell-to-cell communication. In a novel study by Serasanambati et $\mathrm{al}^{27}$ they proved for the first time in reported literature how cancer cells can induce changes in the phenotype of neighboring cells through the upregulation of VOCs. Among other interesting experiments, they co-cultured in an in-vitro fashion normal lung cells and tumoral cells, which shared their headspace but were physically unconnected. At $\mathrm{T}_{48}$ significant cell morphology and apoptotic changes were observed in the normal cells, proving thus that these alterations are affected by volatile signals.

Another unexpected finding when co-culturing two different lung cancer cell lines (physically connected) was that the VOCs concentration detected in their headspace was significantly augmented in comparison to physically unconnected cells. Hence, the authors propose that cancer cells "talk to one another", which further induces changes in the surrounding cell groups.

Noteworthy is that the number of VOCs detected, whose quantity varied significantly, was on average 20 different ones (characterized by GC-MS). While it has been hypothesized how each one can separately induce change, ${ }^{28}$ it is likely that the observed effects are attributed to all of them acting as a whole rather than as single entities.

Much has been written about the benefits of using VOCs as a diagnostic tool in the clinic. We believe that one of its big advantages in contrast to imagenologic screening techniques, that can lead to timely diagnosis, is its threshold for sensing disease. Even when LDCT might be able to screen for small nodules while they are still resectable, amounts below $10^{5}$ cells cannot be seen, ${ }^{29}$ and so carcinogenesis cannot be stopped at its foundation, rather only once there is an established disease.

In contrast, based on its underlying working principle in which minor metabolic pathway alterations can be identified by the change in the VOCs composition, ${ }^{30}$ genetic alterations leading to uncontrolled cellular proliferation can be sensed way before clinical symptoms develop. Moreover, different mutational genotypes can be identified, ${ }^{31}$ and so personalized therapy can be planned ahead of time would it be needed further down the line along the development of the disease. In this fashion, also findings which are too small to undergo histo-pathological sampling can be correctly assessed and the long and uncertain surveillance that happens now-a-days can be avoided.

Worthy of mention is the work published by Peled et $\mathrm{al}^{31}$ in which by reading the volatile "fingerprint" of in vitro cultivated cancer cells with a cross-reactive nanosensor array based on gold nano particles (GNP) they were able to correctly classify 19 cell lines according to EGFRmut, KRASmut, EML4-ALK fusion, or wild type (wt) for the three mutations. With previous GC-MS analysis, they identified over 600 different VOCs in each headspace, but, when performing the comparative studies, they found five distinguishing VOCs that allowed them to properly classify the cell lines according to mutations. In this fashion, they propose not only diagnosing, but more 
importantly monitoring mutational shifts conferring resistance to applied therapies in a timely manner.

Some other advantages of employing VOCs in screening and diagnosis (especially when using a nanosensor) are its non-invasive character, quick results, easy implementation and unexpensive cost, wide availability, small size and portability, high sensitivity, and no technical knowledge required for its use. ${ }^{29}$

\section{VOCs: A Link on the Chain of Early Diagnosis}

As previously mentioned, the benefits of the current screening guidelines with LDCT are contested. Nevertheless, when inclusion criteria are properly established and followed through to screen high risk populations, reductions in mortality of up to a $20 \%$ have been reported. ${ }^{8}$

It is highly probable that, after all, LDCT does have a place in the screening/early detection of lung cancer, but needs to be used in a more refined fashion. By the looks of it, we are currently skipping important testing steps that come before LDCT has any place at all. Furthermore, the National Lung Screening Trial (NLST) on its guidelines establishes protocols for dealing with nodules of $4 \mathrm{~mm}$ and above, ${ }^{32}$ while there is no clear direction on how followup should be performed for findings less than this.

In the natural history of lung cancer, the finding of a nodule might place us already late in the quest for cure. Earlier changes now-a-days can and should be identified.

Seijo et al, ${ }^{16}$ after reviewing all currently available biomarkers, proposed a study model in which a clinically validated biomarker could be incorporated as an independent risk factor from smoking and age to include patients in screening programs with LDCT that currently are at risk but do not meet the used criteria. After assessing for accuracy of methods, better sequences and combinations of testing could start being implemented. In this way they claim that "such a study would pave the way for a lung cancer screening strategy driven or at least reinforced by biomarkers of risk". Moreover, this idea is reinforced by several studies that combine current imagenological techniques with novel biomarkers and have proved that such models "outperform the use of serum biomarkers alone and overall represent a very promising approach for the future of early lung cancer detection, especially if artificial intelligence is incorporated". ${ }^{33,34}$
This is further confirmed by the fact that sensitivity of lung cancer detection with E-noses is the highest in stage I as opposed to later stages, reaching over $90 \%{ }^{35}$

In an age where incidental radiologic findings of a solitary pulmonary nodule (SPN) is on the rise, the prompt and accurate decision of which need to be followed and which represent benign lesions is of extreme importance. As of now, consensus guidelines agree that the management of a SPN is based on imagenological followup, even when there is clear evidence that lesions over $8 \mathrm{~mm}$ confer a $9.7 \%$ probability of lung cancer. ${ }^{36}$ This in turn not only brings with it tremendous costs to the healthcare system, but also high stress and uncertainty to patients who can be up to 5 years in the follow-up of a lesion. In this field, Electronic noses (E-noses) can be of tremendous value, allowing for quicker and sharper sorting of findings, thus benefiting both patients and clinicians alike.

Reports of the last years have underlined the benefit of using novel biomarkers in conjunction with radiological findings to better sort malignant from benign findings. ${ }^{37,38}$

\section{GC-MS versus Electronic Nose: Mimicking Nature}

While GC-MS has been used as the gold standard for recognition of $\mathrm{VOCs}^{39}$ given its ability to precisely identify each compound separately, several downsides have been recognized along its use which make it less attractive as a tool for clinicians to employ.

As mentioned in our previous review, ${ }^{24}$ high complexity of collection techniques and the need for trained personnel makes it difficult to apply in a clinical setting. Moreover, the decline of compounds over time inside the storage bags makes results not always reliable.

To date more than 3,000 VOCs have been identified that could be related to lung cancer. ${ }^{40}$ But, while there might be some that vary more than others in a pathological state, no single VOC can stand as a specific biomarker for disease prediction. Rather, a selection of VOCs needs to be used for enhancing sensitivity.

Good examples of this unspecificity are acetone and isoprene. Apart from being measured in lung cancer, they have been used in numerous other diseases such as cystic fibrosis, ${ }^{41}$ malaria, ${ }^{42}$ renal failure, ${ }^{43}$ epilepsy, ${ }^{44}$ as well as physiological conditions such as aging, gender, and race. ${ }^{45}$

In light of this, it is clear that if we want to be able to implement a simple, reliable, and user-friendly interphase, Electronic noses are the way to go. While not being able to 
identify each compound separately, an E-nose, consisting of a "cross reactive" sensor array and an artificial neural network (ANN), can identify patterns of VOCs, the so-called "breathprint", in an even more sensitive and specific manner. In this fashion, a certain VOC mixture represents a pathological profile rather than a single analyte. This idea follows precisely the modus operandi of the biological nose. ${ }^{46}$

Figure 1 depicts this concept, and shows some of the uses VOC currently have when used for cancer detection.

In living beings with a neural system, smell is identified as a pattern of olfaction in a background of diverse odorants rather than single elements. ${ }^{47}$ After receptor binding in the olfactory epithelium, located apically in the nose, the signal is transported to the olfactory bulb (OB), the first relay station where olfactory information is converted into a spatiotemporal neural code for higherorder information processing. ${ }^{48}$

Peripherally, each olfactory receptor has multiple binding sites for different odorants. This in turn allows for the property of "cross-reactivity." In this mode, activation of different receptors creates different signal patterns, allowing for the recognition of thousands of different smells. ${ }^{49}$ Lastly, the information travels to the olfactory cortex in a direct and parallel fashion for final processing and from there to accompanying subregions such as the thalamus for integrative handling with somatosensitive, gustative, visual, and auditory data. $^{50}$

It is of importance to note that while early studies were focused on detecting single diseases and were not accurate at discerning between different pathological conditions (ie, COPD, ILD, and lung cancer), newer technologies which use machine learning algorithms and employ a wider variety of VOCs for their analysis have managed to discriminate not only between healthy vs pathological samples, but also classify them per pathology with high sensitivity and accuracy. ${ }^{51,52}$

A review of the most common VOCs found in lung cancer goes beyond the spectrum of this review, but can be found elsewhere. ${ }^{53-55}$

\section{Latest Updates \\ PD-LI}

One of the most ground moving and up-to-date studies, published in late 2019, was a prospective observational study in patients receiving immune checkpoint inhibitor therapy for NSCLC (pembrolizumab o nivolumab).

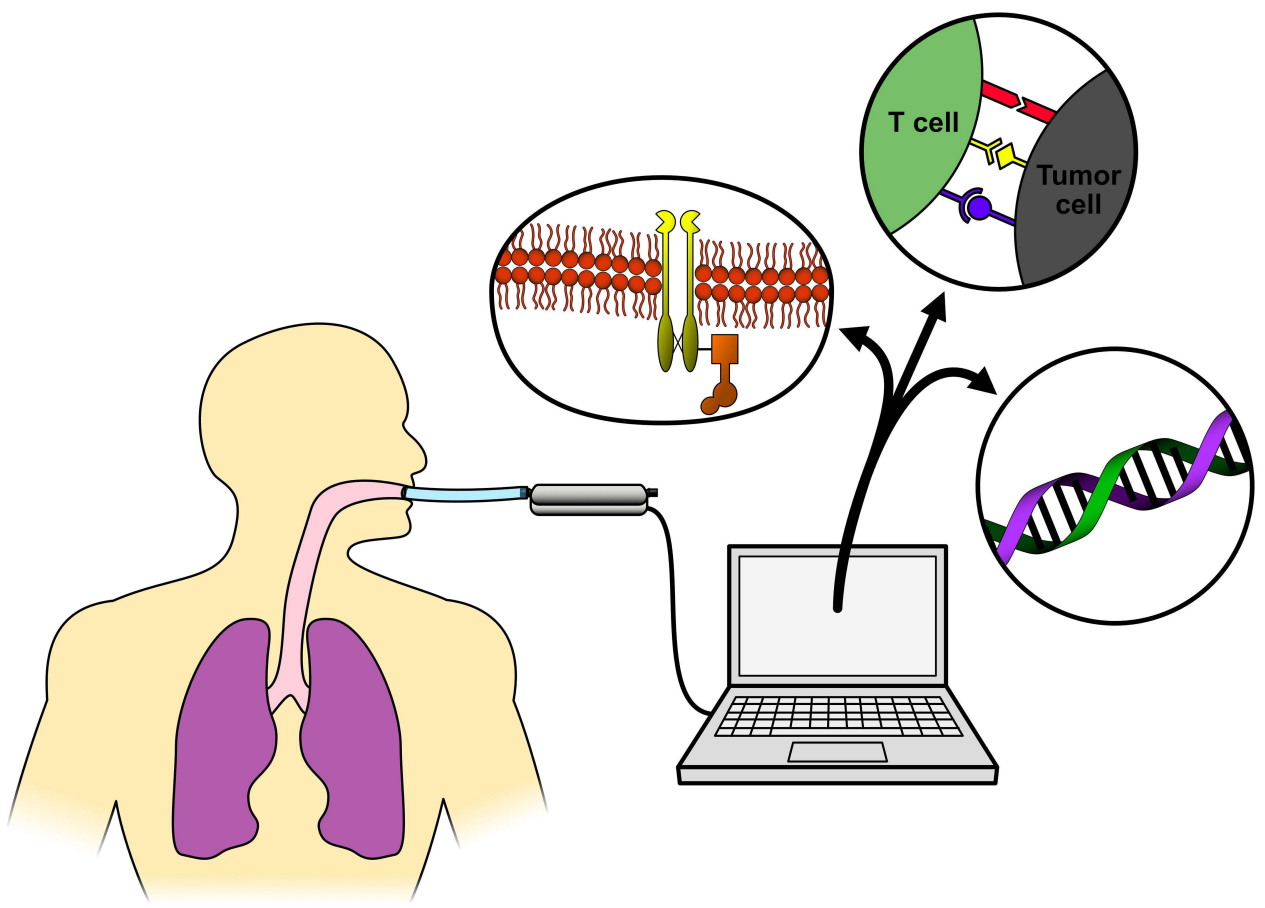

Figure I Breath biopsy.

Notes: Figure I shows how Electric nose technology allows for recognition of early changes in carcinogenesis (ie, EGFR receptor). Specific mutations conferring pathway addiction can be promptly identified. Novel biomarkers such as PD-(L)I can also be identified by E-noses, predicting response to immunotherapy. When working with exhaled breath condensate (EBC) even DNA molecules can be obtained and sequenced. It is likely that this technology will soon be able to complement or even displace invasive biopsies. 
A commercially available E-nose "SpiroNose" which incorporates seven metal oxide semiconductors (MOS) and elements of a spirometer was used. In it, the investigators performed breath tests at baseline and at 3 months of therapy, alongside with PD-L1 biomarker IHC assay kits. The aim was two-fold. First was the distinction between responders and non-responders before the start of therapy, and, second, to compare predictive accuracy between the standard IHC assay and the E-nose for non-responders (to be able to discontinue therapy for those who were not benefiting from it). Given this, and in order not to mistakenly withhold therapy from any patient, they set specificity to $100 \%$ for non-responders.

The results were very promising. For a total of 143 patients, using the difference in signal output of four combined sensors, breath profiles of responders versus non-responders were distinguished with a ROC-AUC curve of 0.89 ( $95 \%$ CI $=0.82-0.96)$. Discriminant analysis showed an accuracy of $82 \%$.

When validating the sensor, a subgroup (validation set) of 51 patients was used. The cut-off set in order to achieve $100 \%$ specificity was 0.72 . With this value, specificity achieved was of $43 \%$. Twelve out of the 51 patients (24\%) were correctly predicted as non-responders (more accurate than the IHC assay). ${ }^{56}$

Even while this was only a small trial, and to the best knowledge of the investigators, the first of its kind, results are promising. First, it proves that susceptibility to PD-L1 therapy is reflected by a particular breath pattern, and, second, that by applying this technology $24 \%$ of patients could potentially be withheld from unnecessary therapy. ${ }^{56}$ Moreover, when biopsy tissue is not available for IHC staining, an E-nose based technology could be applied with at least equally good results.

Interestingly enough, this most likely follows the idea that VOCs are results of intra and intercellular mechanisms related to inflammation, oxidative stress, mutational burden, and overall changes in the metabolic state.

\section{Machine Learning Algorithms}

Another exciting study was published in late 2018 by Tirzite et $\mathrm{al}^{51}$ in which, after separating 475 patients into histologically confirmed cancer vs non-cancer and further into smokers vs non-smokers, they were able to retroactively classify them into categories with over $95 \%$ sensitivity and $90 \%$ specificity. Their claim was that these high percentages were due not only to the E-nose used
(Cyranose 320), but rather to the algorithm used to process data obtained.

Most studies up until now have focused their efforts on building a better and more sensitive device, while most algorithms are manually designed using standard statistical methods. In this study, the use of logistic regression analysis (LRA) was used. As brought by them, self-learning or machine learning algorithms which improve after each reading and adapt to calculations of the incoming data to provide an optimal result are only starting to be used.

Following the same idea, Chi-Hsiang Huang achieved a $92.7 \%$ accuracy in classifying 244 individuals while employing a non-linear support vector machine (SVM), another machine learning technique. ${ }^{57}$

So much so has the application of innovative algorithms enhanced the accuracy of "breathomics" that "the eNose company", 58 a Netherlands based electronic nose supplier includes in its product (an e-nose consisting of three metal oxide sensors: "Aeonose"), a dedicated software package "Aethena" comprising techniques for data pre-treatment, data compression methods, ANN training, and classification.

Remarkably, Kort et $\mathrm{al}^{59}$ reported an improvement from $93.5 \%$ to $95.7 \%$ in sensitivity for distinguishing NSCLC patients from controls when applying a multivariate logistic regression model to records obtained using the trained ANN with data obtained from the E-nose "Aeonose".

\section{Integrative Technologies}

In an attempt to enhance sensitivity and specificity, typedifferent sensor arrays have been built and tested in the last couple of years. In this modality, two or more different E-nose techniques are mixed into one single array (an explanation of the different types of sensors goes beyond the scope of this review and can be found elsewhere in a detailed fashion). ${ }^{28}$ Notably, in $2017 \mathrm{Li}$ et al ${ }^{60}$ put together 14 gas sensors and two temperature/humidity sensors. Out of them, eight were MOS, four electrochemical gas, one Hotwire gas, and one catalytic combustion type. Five algorithms were used in interpreting the cross-referenced data. An outstanding $91.58 \%$ and $91.72 \%$ of sensitivity and specificity, respectively, were reached when separating 47 samples into positive and negative for lung cancer.

Pardo et $\mathrm{al}^{61}$ concluded that the performance achieved by a hybrid array is superior to the one of the top chosen sensor subsets for any single sensor type, these constantly outperform single transducer arrays. ${ }^{62}$ 
After an in-depth analysis of the E-nose technology in all of its current applications, Broza et $\mathrm{al}^{28}$ claimed that "arguably, the hybrid approach is indeed the next promising step of these sensors".

\section{External Probe VOCs}

VOCs are separated into endogenous and exogenous. Endogenous VOCs are compounds that result from internal cell metabolism, physiologic, and pathologic processes. Exogenous are the ones that are inhaled and may or may not undergo changes in the respiratory process. They derive from what is termed "environmental exposure". Historically, the first ones have been of interest while the second ones have been seen as contaminants and termed "confounders". So much so that the "alveolar gradient" technique has been developed, in which the inhaled fraction is subtracted from the exhaled in order to eliminate environmental exposure and get a more accurate reading. ${ }^{63}$

An innovative approach that has been proposed lately in order to eliminate this issue is the use of an external probe VOC. In this method, an exogenous substrate is given to the subject and then measured when exhaled. This is to investigate the metabolic activity or microbiome in relation to health and disease ${ }^{64}$ Classic examples are the Urea breath test (UBT) test for rapid H. pylori detection ${ }^{65}$ and the Hydrogen breath test (HBT) for detection of malabsorption and small intestine bacterial overgrowth (SIBO) ${ }^{66}$

The underlying reasoning for cancer detection would be that the high cellular metabolism and elevated mitotic rate can be used to indicate the presence of pathology, resulting in a specific measurable metabolite independent of other VOCs that can alter results. A model of this, while not in the breath area, is the use of the FDG-PET in which the upregulation of the GLUT1 transporter is exploited for better detection of cancer foci. External probes for cancer are not yet a reality but are a promising technique that would make VOC standardization easier to achieve.

\section{Ongoing Clinical Trials}

As previously explained, GC-MS has important downsides, mainly due to its collection techniques and clinical practice incompatibility. A respiration collector for in vitro analysis (ReCIVAC) ${ }^{67}$ has been developed by Owlstone Medical, commissioned by the British NHS, that solves these issues to an extent. Currently being used in an active interventional clinical trial (Lung cancer indicator detection: "LuCID") ${ }^{68}$ involving over 25 clinical sites in five countries and 4,000 patients, it consists of a hand-held machine, made available in doctors practices, in which patients breathe for a continuous 10 minutes onto the sampler. Instead of the commonly used Tedlar bags for breath storage, it uses thermal desorption adsorbent tubes which allow for a more consistent concentration of gases and less decay over time. Furthermore, in order to catch alveolar breath and not airway dead space, it employs real-time exhaled $\mathrm{CO}_{2}$ measurements to estimate the portion (lower airway, upper airway or alveolar) of breath entering the device. Breath is sampled at the appropriate $\mathrm{CO}_{2}$ levels via active pumps directly onto four built-in adsorbent tubes. Technology wise, it couples field asymmetric-waveform ion-mobility spectrometry (FAIMS) to the classic GC-MS which allows for better analyte fractionation and less contamination disturbance. Having reached its first completion date in December 2020, the full study is expected to provide final results in December 2022. The primary outcome will be determining the Area Under the Curve (AUC) for the used algorithm with optimal point sensitivity, specificity, positive and negative predictive values. If proved successful, it could be the first clinically available point-of-care breath sampler. In this way, the $\operatorname{ReCIVA}^{\odot}$ is a large step toward reaching a standard in exhaled breath sampling and bringing it closer to the physicians practice.

\section{Wide Spectrum of Use}

As exposed along the review, E-Noses have been put to use not only in early diagnosis, rather also, they have been implemented in every stage along the developing disease, as Figure 2 shows. This, in turn, makes E-Noses an even more promising tool given their versatility. Still missing in the medical world today, is a device that can be employed as a diagnostic tool, provide pathological information, serve as a follow-up and surveillance instrument, and also determine staging and response to therapy. Table 1 shows a handful of selected published papers where this can be visually appreciated.

Accurately used, it could provide a compliment or replacement not only for LDCT but rather to pathology services and follow-up CAT scans.

\section{Current Limitations}

While as we have seen E-noses have multiple benefits, there are still some challenges around this technology that have not yet been completely solved. Zhang et al brings them in a very clear fashion in his e-book "E-Nose Algorithms and Challenges. ${ }^{69}$ They define them as the 3D: discreteness, drift, and disturbance. 


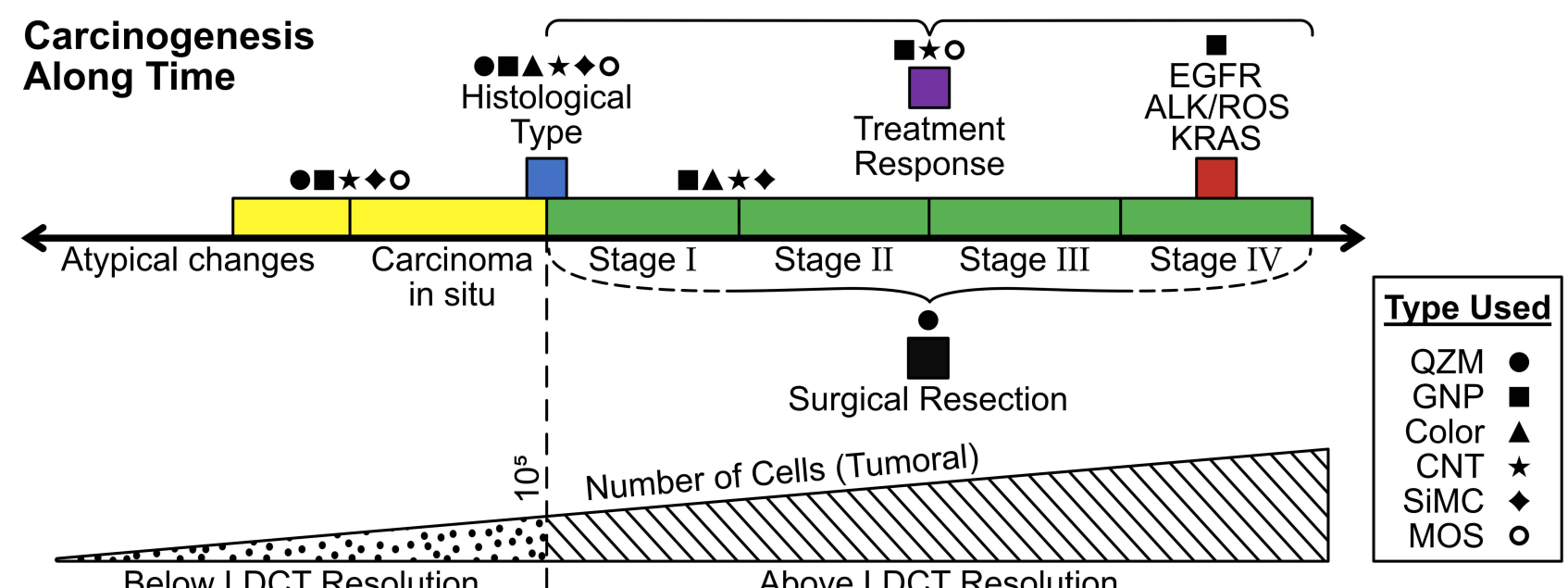

Figure 2 Current E-Nose applications in cancer.

Notes: Figure 2 shows the applications E-noses have had along the last decades in every carcinogenesis stage. Symbols represent technologies of E-Noses used in as per the studies brought in Table I. Colors are well matched to indicate the step where its use is made.

Discreteness deals with reproducibility of outputs using the same sensor arrays. Even when manufactured in a duplicable fashion, there might be a slight variability in the results obtained, such that under equal sensing conditions the output will be different (signal shift). This is due to resistance and sensitivity discrepancies among identical E-Nose arrays given inherent material variability.

Drift is the innate decay of material along time that inevitably happens due to several factors such as humidity, temperature, pressure, aging, etc. This does not allow for long-term stable use. Results will inevitably vary with the passing of time, even when exposed to the same VOCs. While drift effect can be learned and predicted, still, identical sensors can show different drift effects given environmental and usage conditions.

Last, and probably the biggest VOC and E-nose challenge to date, is disturbance. Disturbance is the interference caused by the "non-target" VOCs. As an example, if a determined assay has detectors for benzene, octane, carbon monoxide, and ammonia, any other gas would be viewed as disturbance and can significantly alter measurements. While there are solutions for this in the sampling process such as collecting a baseline and then using a "disturbance exhaust" to get rid of non-desired VOCs, a process known as "disturbance recognition and elimination", still, this can only deal with a few nontarget gases, while it is known that in real life there could be thousands of disturbances. Since VOCs first description in 1971 by Pauling et al., ${ }^{70}$ this issue has been raised. They clearly express: "In order that the results of the analysis be significantly representative, it is essential that it be standardized with respect to diet and other environmental influences, such as the nature of the intestinal flora." These unresolved challenges, for the most, have led to the inability to standardize VOCs and have thus kept them away from everyday clinical practice. ${ }^{71,72}$

\section{EBC: A Sister Technology}

Comprising the exhaled breath, together with the VOCs which have been the focus of our review, are the NonVolatile organic compounds, better known as the exhaled breath condensate (EBC). This is a term coined for the first time in 1913 by a German bacteriologist when describing "the presence of an organic body in expired air", ${ }^{73}$ the study of the non-volatile part of breath has gained much attention in the last years.

After breathing into a cool condenser, a liquid phase of breath is collected. It consists of mostly water vapor and aerosolized droplets of airway lining fluid (ALF). This in turn contains an endless number of molecules such as DNAs, RNAs, proteins, and metabolites from the respiratory tract. As well, water soluble VOCs such as carbon dioxide, nitric oxide, and ethane can also be detected. ${ }^{74}$ One of its most interesting applications is the possibility of obtaining tumor DNA in a non-invasive manner. ${ }^{75,76}$ This may represent a novel technique for being able to run tumor DNA testing while not having to recur to invasive procedures.

While beyond the subject of this review, multiple studies have successfully sequenced EBC DNA, not only by classic methods after PCR amplification, but also through NGS. ${ }^{77}$ Investigators in a series of different $\mathrm{EBC}$ based studies were able to find TP53 mutations, ${ }^{78} \mathrm{EGFR},{ }^{79} \mathrm{CDKN} 2 \mathrm{~A},{ }^{80} \mathrm{KRAS},{ }^{81}$ 


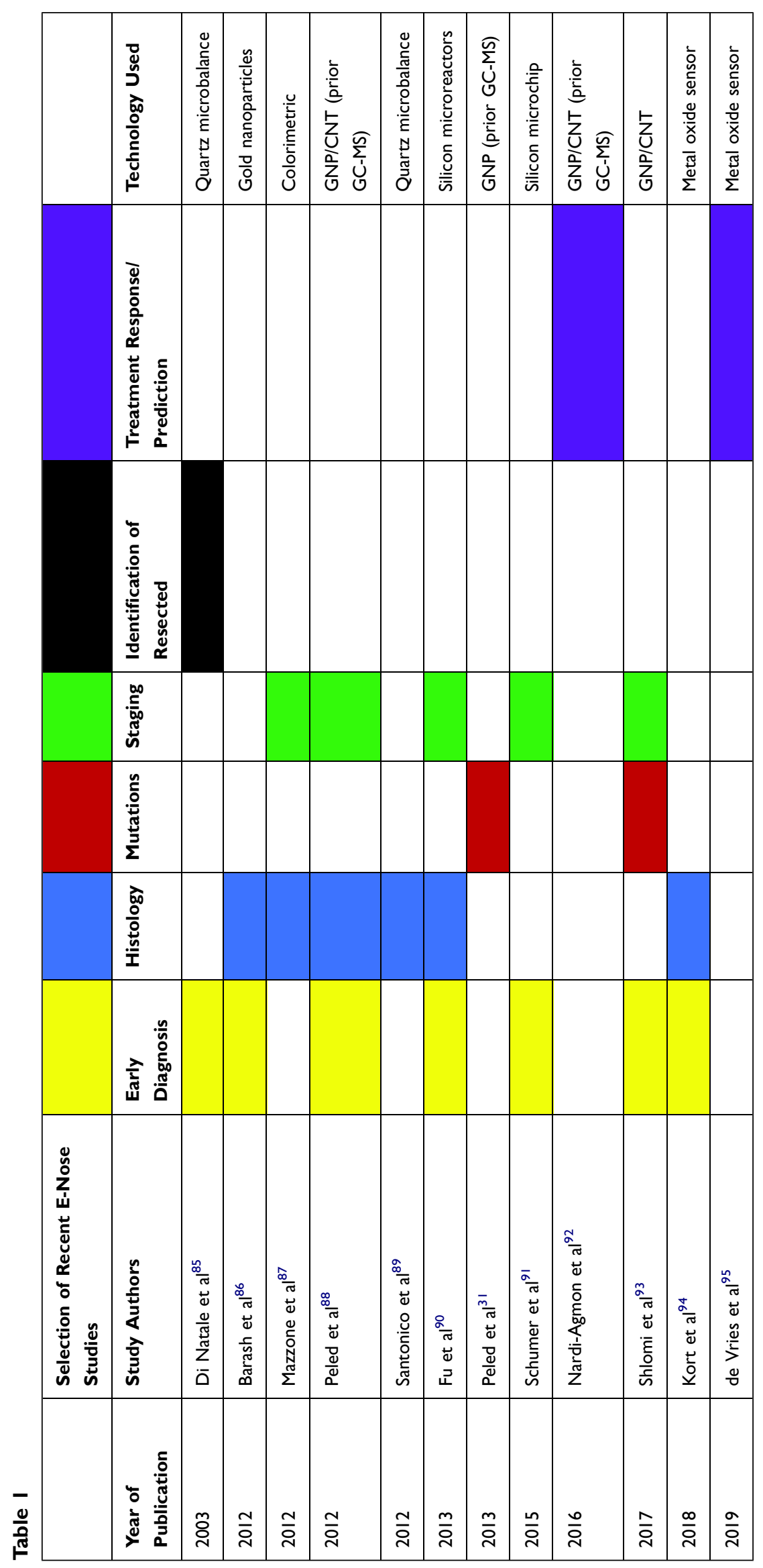


EGFR T790M resistance mutation, ${ }^{82}$ and microsatellite instability ${ }^{83}$ among others.

While this technology can possibly provide even further or maybe complimentary information to VOCs, the current issues surrounding it are the same. No clinical instrument has been established due to lack or standardization and establishment of clinical values. ${ }^{84}$

\section{Final Remarks}

- Opportune screening and earlier diagnosis of lung cancer holds the key for lowering mortality.

- VOCs take part in establishing carcinogenesis and building the tumor microenvironment (TME).

- VOCs are a promising technology in ongoing development that can complement current screening techniques for higher detection rates and timely diagnosis.

- By replicating the olfactory system, E-noses can identify smell patterns leading to higher sensibility and sensitivity than single analytes.

- Response to therapy and treatment resistant mutational changes can be successfully monitored in a non-invasive manner.

- Further understanding of the VOCs origins in cellular metabolism and role in carcinogenesis is fundamental for advancing the technology.

- Challenges such as standardization still need to be addressed in order to reach clinical validation.

- Clinical trials are underway to take breath sampling tools to the clinician hands in the upcoming years.

\section{Disclosure}

Nir Peled is Advisor \& Honorarium from Research with AstraZeneca, Bayer, Boehringer Ingelheim, Bristol-Myers Squibb, Eli Lilly, FoundationMedicine, Guardant360, Merck, MSD, Novartis, NovellusDx, Pfizer, Roche, Takeda, and holds the following international patents (IP): Volatile Organic Compounds For Detecting Cell Dysplasia and Genetic Alterations Associated with Lung Cancer; WO2012023138 Breath Analysis of Pulmonary Nodules. US20130150261 A1. Apparatus for treating a target site of a body; WO/2015/ 059646. The rest of the authors hold no conflict of interest.

\section{References}

1. Cancer. Available from: https://www.who.int/news-room/fact-sheets /detail/cancer. Accessed January 16, 2021.

2. CDC. An update on cancer deaths in the United States. Available from: https:/www.cdc.gov/cancer/dcpc/research/update-on-cancer-deaths /index.htm. Accessed January 16, 2021.
3. About lung cancer. Available from: https://www.cancer.org/cancer/ lung-cancer/about/key-statistics.html. Accessed January 17, 2021.

4. Walser T, Cui X, Yanagawa J, et al. Smoking and lung cancer: the role of inflammation. Proc Am Thorac Soc. 2008;5(8):811-815. doi:10.1513/pats.200809-100TH

5. Wood DE, Kazerooni EA, Aberle D, et al. NCCN guidelines version 1.2021 lung cancer screening version $1 ; 2021$. Available from: https:// www.nccn.org/professionals/physician_gls/pdf/lung_screening.pdf. Accessed January 17, 2021.

6. Usman Ali M, Miller J, Peirson L, et al. Screening for lung cancer: a systematic review and meta-analysis. Prev Med. 2016;89:301-314. doi:10.1016/j.ypmed.2016.04.015

7. Nemesure B, Plank A, Reagan L, Albano D, Reiter M, Bilfinger TV. Evaluating efficacy of current lung cancer screening guidelines. J Med Screen. 2017;24(4):208-213. doi:10.1177/ 0969141316689111

8. National Lung Screening Trial Research Team. Reduced lung-cancer mortality with low-dose computed tomographic screening. $N$ Engl J Med. 2011;365(5):395-409. doi:10.1056/nejmoa1102873

9. O'Dowd EL, Baldwin DR. Lung cancer screening-low dose cT for lung cancer screening: recent trial results and next steps. Br J Radiol. 2018;91:1090. doi:10.1259/bjr.20170460

10. Kathuria H, Gesthalter Y, Spira A, Brody JS, Steiling K. Updates and controversies in the rapidly evolving field of lung cancer screening, early detection, and chemoprevention. Cancers. 2014;6 (2):1157-1179. doi:10.3390/cancers6021157

11. WHO position paper on mammography screening. Available from: https://www.paho.org/hq/dmdocuments/2015/WHO-ENGMammography-Factsheet.pdf. Accessed January 16, 2021.

12. Singh H, Nugent Z, Demers AA, Kliewer EV, Mahmud SM, Bernstein $\mathrm{CN}$. The reduction in colorectal cancer mortality after colonoscopy varies by site of the cancer. Gastroenterology. 2010;139(4):1128-1137. doi:10.1053/j.gastro.2010.06.052

13. Pan J, Xin L, Ma YF, Hu LH, Li ZS. Colonoscopy reduces colorectal cancer incidence and mortality in patients with non-malignant findings: a meta-analysis. Am J Gastroenterol. 2016;111(3):355-365. doi:10.1038/ajg.2015.418

14. Hakama M, Moss SM, Stenman UH, et al. Design-corrected variation by centre in mortality reduction in the ERSPC randomised prostate cancer screening trial. J Med Screen. 2017;24(2):98-103. doi:10.1177/0969141316652174

15. Wood DE, Kazerooni EA, Baum SL, et al. Lung cancer screening, version 3.2018, NCCN clinical practice guidelines in oncology. $J$ Natl Compr Canc Netw. 2018;16:412-441. doi:10.6004/ jncen.2018.0020

16. Seijo LM, Peled N, Ajona D, et al. Biomarkers in lung cancer screening: achievements, promises, and challenges. J Thorac Oncol. 2019;14(3):343-357. doi:10.1016/j.jtho.2018.11.023

17. Rolfo C, Russo A. Liquid biopsy for early stage lung cancer moves ever closer. Nat Rev Clin Oncol. 2020;17(9):523-524. doi:10.1038/ s41571-020-0393-z

18. Asakura K, Kadota T, Matsuzaki J, et al. A miRNA-based diagnostic model predicts resectable lung cancer in humans with high accuracy. Commun Biol. 2020;3(1):1-9. doi:10.1038/s42003-020-0863-y

19. Fujiwara K, Fujimoto N, Tabata M, et al. Identification of epigenetic aberrant promoter methylation in serum DNA is useful for early detection of lung cancer. Clin Cancer Res. 2005;11(3):1219-1225.

20. Billatos E, Vick JL, Lenburg ME, Spira AE. The airway transcriptome as a biomarker for early lung cancer detection. Clin Cancer Res. 2018;24(13):2984-2992. doi:10.1158/1078-0432.CCR-16-3187

21. Yang B, Li X, Ren T, Yin Y. Autoantibodies as diagnostic biomarkers for lung cancer: a systematic review. Cell Death Discov. 2019;5 (1):1-5. doi:10.1038/s41420-019-0207-1

22. Mansurova M, Ebert BE, Blank LM, Ibáñez AJ. A breath of information: the volatilome. Curr Genet. 2018;64(4):959-964. doi:10.1007/ s00294-017-0800-X 
23. Anand SS, Philip BK, Mehendale HM. Volatile organic compounds. In: Encyclopedia of Toxicology, 3rd ed. Elsevier; 2014:967-970. doi:10.1016/B978-0-12-386454-3.00358-4

24. Nardi-Agmon I, Peled N. Exhaled breath analysis for the early detection of lung cancer: recent developments and future prospects. Lung Cancer Targets Ther. 2017;8:31-38. doi:10.2147/LCTT.S104205

25. Janssens E, van Meerbeeck JP, Lamote K. Volatile organic compounds in human matrices as lung cancer biomarkers: a systematic review. Crit Rev Oncol Hematol. 2020;153:103037. doi:10.1016/j. critrevonc.2020.103037

26. D'Alessandro M. Assessing the importance of specific volatile organic compounds in multitrophic interactions; 2006. Available from: http://doc.rero.ch/record/6468. Accessed July 20, 2021.

27. Serasanambati M, Broza YY, Haick H. Volatile compounds are involved in cellular crosstalk and upregulation. Adv Biosyst. 2019;3 (10):1900131. doi:10.1002/adbi.201900131

28. Broza YY, Vishinkin R, Barash O, Nakhleh MK, Haick H. Synergy between nanomaterials and volatile organic compounds for non-invasive medical evaluation. Chem Soc Rev. 2018;47 (13):4781-4859. doi:10.1039/c8cs00317c

29. Fischer BM, Olsen MWB, Ley CD, et al. How few cancer cells can be detected by positron emission tomography? A frequent question addressed by an in vitro study. Eur J Nucl Med Mol Imag. 2006;33 (6):697-702. doi:10.1007/s00259-005-0038-6

30. Hakim M, Broza YY, Barash O, et al. Volatile organic compounds of lung cancer and possible biochemical pathways. Chem Rev. 2012;112 (11):5949-5966. doi:10.1021/cr300174a

31. Peled N, Barash O, Tisch U, et al. Volatile fingerprints of cancer specific genetic mutations. Nanomed Nanotechnol Biol Med. 2013;9 (6):758-766. doi:10.1016/j.nano.2013.01.008

32. Team TNLSTR. Results of initial low-dose computed tomographic screening for lung cancer. $N$ Engl J Med. 2013;368(21):1980-1991. doi:10.1056/nejmoa1209120

33. Jiang R, Dong X, Zhu W, et al. Combining PET/CT with serum tumor markers to improve the evaluation of histological type of suspicious lung cancers. PLoS One. 2017;12(9):e0184338. doi:10.1371/journal.pone. 0184338

34. Broodman I, Lindemans J, Van Sten J, Bischoff R, Luider T. Serum protein markers for the early detection of lung cancer: a focus on autoantibodies. J Proteome Res. 2017;16(1):3-13. doi:10.1021/acs. jproteome.6b00559

35. Gasparri R, Santonico M, Valentini C, et al. Volatile signature for the early diagnosis of lung cancer. $J$ Breath Res. 2016;10(1):016007. doi:10.1088/1752-7155/10/1/016007

36. Loverdos K, Fotiadis A, Kontogianni C, Iliopoulou M, Gaga M. Lung nodules: a comprehensive review on current approach and management. Ann Thorac Med. 2019;14(4):226. doi:10.4103/ATM. ATM_110_19

37. Liang W, Zhao Y, Huang W, et al. Non-invasive diagnosis of early-stage lung cancer using high-throughput targeted DNA methylation sequencing of circulating tumor DNA (ctDNA). Theranostics. 2019;9(7):2056-2070. doi:10.7150/THNO.28119

38. Lin Y, Leng Q, Jiang Z, et al. A classifier integrating plasma biomarkers and radiological characteristics for distinguishing malignant from benign pulmonary nodules. Int $J$ Cancer. 2017;141 (6):1240-1248. doi:10.1002/IJC.30822

39. Davis MD, Fowler SJ, Montpetit AJ. Exhaled breath testing - a tool for the clinician and researcher. Paediatr Respir Rev. 2019;29:37-41. doi:10.1016/j.prrv.2018.05.002

40. Phillips M, Gleeson K, Hughes JMB, et al. Volatile organic compounds in breath as markers of lung cancer: a cross-sectional study. Lancet. 1999;353(9168):1930-1933. doi:10.1016/S0140-6736(98)07552-7

41. Barker M, Hengst M, Schmid J, Buers H-J, Klemp D, Koppmann R. Volatile organic compounds in the exhaled breath of young patients with cystic fibrosis. Eur Respir J. 2006;27:929-936. doi:10.1183/ 09031936.06.00085105
42. Berna AZ, McCarthy JS, Wang RX, et al. Analysis of breath specimens for biomarkers of plasmodium falciparum infection. $J$ Infect Dis. 2015;212(7):1120-1128. doi:10.1093/infdis/jiv176

43. Davies S, Španel P, Smith D. A new "Oonline" method to measure increased exhaled isoprene in end-stage renal failure. Nephrol Dial Transplant. 2001;16(4):836-839. doi:10.1093/ndt/16.4.836

44. Musa-Veloso K, Likhodii SS, Rarama E, et al. Breath acetone predicts plasma ketone bodies in children with epilepsy on a ketogenic diet. Nutrition. 2006;22(1):1-8. doi:10.1016/j.nut.2005.04.008

45. Ruzsányi V, Kalapos MP. Breath acetone as a potential marker in clinical practice. J Breath Res. 2017;11(2):024002. doi:10.1088/ 1752-7163/aa66d3

46. Wasilewski T, Gębicki J, Kamysz W. Bioelectronic nose: current status and perspectives. Biosens Bioelectron. 2017;87:480-494. doi:10.1016/j.bios.2016.08.080

47. Benjaminsson S, Herman P, Lansner A. Performance of a computational model of the mammalian olfactory system. Neuromorphic Olfaction. 2016:173-211. doi:10.1201/b14670-11

48. Imai T. Construction of functional neuronal circuitry in the olfactory bulb. Semin Cell Dev Biol. 2014;35:180-188. doi:10.1016/j. semcdb.2014.07.012

49. Fitzgerald J, Fenniri H. Cutting edge methods for non-invasive disease diagnosis using e-tongue and e-nose devices. Biosensors. 2017;7 (4):59. doi:10.3390/bios7040059

50. Zhou G, Lane G, Cooper SL, Kahnt T, Zelano C. Characterizing functional pathways of the human olfactory system. Elife. 2019;8: e47177. doi:10.7554/eLife.47177

51. Tirzite M, Bukovskis M, Strazda G, Jurka N, Taivans I. Detection of lung cancer with electronic nose and logistic regression analysis. $J$ Breath Res. 2019;13(1):16006. doi:10.1088/1752-7163/aae1b8

52. Rodríguez-Aguilar M, Díaz de León-martínez L, Gorocica-Rosete P, et al. Application of chemoresistive gas sensors and chemometric analysis to differentiate the fingerprints of global volatile organic compounds from diseases. Preliminary results of COPD, lung cancer and breast cancer. Clin Chim Acta. 2021;518:83-92. doi:10.1016/J. CCA.2021.03.016

53. Kuo TC, Tan CE, Wang SY, et al. Human breathomics database. Database. 2020;2020. doi:10.1093/database/baz139

54. Janfaza S, Khorsand B, Nikkhah M, Zahiri J. Digging deeper into volatile organic compounds associated with cancer. Biol Methods Protoc. 2019;4(1):bpz014. doi:10.1093/biomethods/bpz014

55. Jalal AH, Alam F, Roychoudhury S, Umasankar Y, Pala N, Bhansali S. Prospects and challenges of volatile organic compound sensors in human healthcare. Acs Sensors. 2018;3:1246-1263. doi: $10.1021 /$ acssensors. 8 b00400

56. Abbasi J. "Electronic nose" predicts immunotherapy response. JAMA. 2019;322(18):1756. doi:10.1001/jama.2019.18225

57. Huang $\mathrm{CH}$, Zeng $\mathrm{C}$, Wang $\mathrm{YC}$, et al. A study of diagnostic accuracy using a chemical sensor array and a machine learning technique to detect lung cancer. Sensors. 2018;18(9):2845. doi:10.3390/ s18092845

58. Specialists in artificial olfaction. Available from: https://www.enose. $\mathrm{nl} /$. Accessed January 30, 2021.

59. Kort S, Brusse-Keizer M, Gerritsen JW, et al. Improving lung cancer diagnosis by combining exhaled-breath data and clinical parameters. ERJ Open Res. 2020;6(1):00221-02019. doi:10.1183/ 23120541.00221-2019

60. Li W, Liu H, Xie D, He Z, Pi X. Lung cancer screening based on type-different sensor arrays. Sci Rep. 2017;7(1):1-12. doi:10.1038/ s41598-017-02154-9

61. Pardo M, Kwong LG, Sberveglieri G, et al. Data analysis for a hybrid sensor array. Sens Actuators B Chem. 2005;106(1):136-143. doi:10.1016/j.snb.2004.05.045

62. Jin C, Kurzawski P, Hierlemann A, Zellers ET. Evaluation of multitransducer arrays for the determination of organic vapor mixtures. Anal Chem. 2008;80(1):227-236. doi:10.1021/ac0715120 
63. Pleil JD, Stiegel MA, Risby TH. Clinical breath analysis: discriminating between human endogenous compounds and exogenous (environmental) chemical confounders. J Breath Res. 2013;7 (1):017107. doi:10.1088/1752-7155/7/1/017107

64. Gaude E, Nakhleh MK, Patassini S, et al. Targeted breath analysis: exogenous volatile organic compounds (EVOC) as metabolic pathway-specific probes. $J$ Breath Res. 2019;13(3):32001. doi:10.1088/1752-7163/ab1789

65. Pathak CM, Bhasin DK, Khanduja KL. Urea breath test for Helicobacter pylori detection: present status. Trop Gastroenterol. 2004;25(4):156-161.

66. Modak AS. Stable isotope breath tests in clinical medicine: a review. $J$ Breath Res. 2007;1:014003. doi:10.1088/1752-7155/1/1/014003

67. ReCIVA ${ }^{\circledR}$ breath sampler. Available from: https://www.owlstonemedi cal.com/products/reciva/?utm_source=breath-sampler-keywords-zone -1\&utm_medium=ga-search\&utm_campaign=00438-breath-samplersearch-breath-sampler-keywords-ga-search-zone-1-20171214\&gclid= CjwKCAiA14WABhAJEiwATUnEF9OL7WEJ4NYAT6pF2VxmcVeIAdm640t3Ak8ub0F5weZHlecD-CirxoCTH4QAvD_BwE. Accessed January 30, 2021.

68. ClinicalTrials.gov. Lung cancer indicator detection - full text view. Available from: https:/clinicaltrials.gov/ct2/show/NCT02612532. Accessed January 30, 2021.

69. Zhang L, Tian F, Zhang D, Zhang L, Tian F, Zhang D. E-nose algorithms and challenges. In: Electronic Nose: Algorithmic Challenges. Singapore: Springer; 2018:11-20. doi:10.1007/978-98113-2167-2_2

70. Pauling L, Robinson AB, Teranishit R, Cary P. Quantitative analysis of urine vapor and breath by gas-liquid partition chromatography. Proc Natl Acad Sci. 1971;68:2374-2376. doi:10.1073/ pnas.68.10.2374

71. Hanna GB, Boshier PR, Markar SR, Romano A. Accuracy and methodologic challenges of volatile organic compound-based exhaled breath tests for cancer diagnosis: a systematic review and meta-analysis. JAMA Oncol. 2019;5(1):1-11. doi:10.1001/ jamaoncol.2018.2815

72. Jia Z, Patra A, Kutty VK, Venkatesan T. Critical review of volatile organic compound analysis in breath and in vitro cell culture for detection of lung cancer. Metabolites. 2019;9(3):52. doi:10.3390/ metabo9030052

73. Amoss HL. Organic matter in the expired breath with especial reference to its inhibiting power on oxidizing ferments. $J$ Exp Med. 1913;17(2):132-151. doi:10.1084/jem.17.2.132

74. Maniscalco M, Fuschillo S, Paris D, Cutignano A, Sanduzzi A, Motta A. Clinical metabolomics of exhaled breath condensate in chronic respiratory diseases. Adv Clin Chem. 2019;88:121-149. doi:10.1016/bs.acc.2018.10.002

75. Kordiak J, Szemraj J, Grabska-Kobylecka I, et al. Intratumor heterogeneity and tissue distribution of KRAS mutation in non-small cell lung cancer: implications for detection of mutated KRAS oncogene in exhaled breath condensate. J Cancer Res Clin Oncol. 2019;145 (1):241-251. doi:10.1007/s00432-018-2779-1

76. Youssef O, Sarhadi VK, Armengol G, Piirilä P, Knuuttila A, Knuutila S. Exhaled breath condensate as a source of biomarkers for lung carcinomas. A focus on genetic and epigenetic markers-A mini-review. Genes Chromosom Cancer. 2016;55(12):905-914. doi:10.1002/gcc.22399

77. Youssef O, Knuuttila A, Piirilä P, Böhling T, Sarhadi V, Knuutila S. Presence of cancer-associated mutations in exhaled breath condensates of healthy individuals by next generation sequencing. Oncotarget. 2017;8(11):18166-18176. doi:10.18632/ oncotarget. 15233

78. Gessner C, Kuhn H, Toepfer K, Hammerschmidt S, Schauer J, Wirtz H. Detection of p53 gene mutations in exhaled breath condensate of non-small cell lung cancer patients. Lung Cancer. 2004;43 (2):215-222. doi:10.1016/j.lungcan.2003.08.034
79. Zhang D, Takigawa N, Ochi N, et al. Detection of the EGFR mutation in exhaled breath condensate from a heavy smoker with squamous cell carcinoma of the lung. Lung Cancer. 2011;73(3):379-380. doi:10.1016/j.lungcan.2011.05.018

80. Chen JL, Chen JR, Huang FF, Tao GH, Zhou F, Tao YJ. Analysis of p16 gene mutations and their expression using exhaled breath condensate in non-small-cell lung cancer. Oncol Lett. 2015;10 (3):1477-1480. doi:10.3892/ol.2015.3426

81. Kordiak J, Szemraj J, Hamara K, Bialasiewicz P, Nowak D. Complete surgical resection of lung tumor decreases exhalation of mutated KRAS oncogene. Respir Med. 2012;106(9):1293-1300. doi:10.1016/j.rmed.2012.06.019

82. Smyth RJ, Toomey SM, Sartori A, et al. Brief report on the detection of the EGFR T790M mutation in exhaled breath condensate from lung cancer patients. J Thorac Oncol. 2018;13(8):1213-1216. doi:10.1016/j.jtho.2018.04.033

83. Carpagnano GE, Lacedonia D, Crisetti E, Martinelli D, FoschinoBarbaro MP. New panel of microsatellite alterations detectable in the EBC for lung cancer prognosis. $J$ Cancer. 2016;7(15):2266-2269. doi: $10.7150 /$ jca. 15921

84. Horváth I, Barnes PJ, Loukides S, et al. A european respiratory society technical standard: exhaled biomarkers in lung disease. Eur Respir J. 2017;49(4):1600965. doi:10.1183/13993003.00965-2016

85. Di Natale C, Macagnano A, Martinelli E, et al. Lung cancer identification by the analysis of breath by means of an array of non-selective gas sensors. Biosens Bioelectron. 2003;18 (10):1209-1218. doi:10.1016/S0956-5663(03)00086-1

86. Barash O, Peled N, Tisch U, Bunn PA, Hirsch FR, Haick H. Classification of lung cancer histology by gold nanoparticle sensors. Nanomedicine. 2012;8(5):580-589. doi:10.1016/j.nano.2011.10.001

87. Mazzone PJ, Wang X-F, Xu Y, et al. Exhaled breath analysis with a colorimetric sensor array for the identification and characterization of lung cancer. J Thorac Oncol. 2012;7(1):137-142. doi:10.1097/ JTO.0b013e318233d80f

88. Peled N, Hakim M, Bunn PA, et al. Non-invasive breath analysis of pulmonary nodules. $J$ Thorac Oncol. 2012;7(10):1528-1533. doi:10.1097/JTO.0b013e3182637d5f

89. Santonico M, Lucantoni G, Pennazza G, et al. In situ detection of lung cancer volatile fingerprints using bronchoscopic air-sampling. Lung Cancer. 2012;77(1):46-50. doi:10.1016/j.lungcan.2011.12.010

90. Fu X-A, Li M, Knipp RJ, Nantz MH, Bousamra M. Noninvasive detection of lung cancer using exhaled breath. Cancer Med. 2014;3 (1):174-181. doi:10.1002/cam4.162

91. Schumer EM, Trivedi JR, van Berkel V, et al. High sensitivity for lung cancer detection using analysis of exhaled carbonyl compounds. $J$ Thorac Cardiovasc Surg. 2015;150(6):1517-1522. doi:10.1016/j. jtcvs.2015.08.092

92. Nardi-Agmon I, Abud-Hawa M, Liran O, et al. Exhaled breath analysis for monitoring response to treatment in advanced lung cancer. $J$ Thorac Oncol. 2016;11(6):827-837. doi:10.1016/j. jtho.2016.02.017

93. Shlomi D, Abud M, Liran O, et al. Detection of lung cancer and EGFR mutation by electronic nose system. J Thorac Oncol. 2017;12 (10):1544-1551. doi:10.1016/j.jtho.2017.06.073

94. Kort S, Tiggeloven MM, Brusse-Keizer M, et al. Multi-centre prospective study on diagnosing subtypes of lung cancer by exhaled-breath analysis. Lung Cancer. 2018;125:223-229. doi:10.1016/j.lungcan.2018.09.022

95. de Vries R, Muller M, van der Noort V, et al. Prediction of response to anti-PD-1 therapy in patients with non-small-cell lung cancer by electronic nose analysis of exhaled breath. Ann Oncol. 2019;30:1660-1666. doi:10.1093/annonc/mdz279 


\section{Publish your work in this journal}

Lung Cancer: Targets and Therapy is an international, peerreviewed, open access journal focusing on lung cancer research, identification of therapeutic targets and the optimal use of preventative and integrated treatment interventions to achieve improved outcomes, enhanced survival and quality of life for the cancer patient. Specific topics covered in the journal include: Epidemiology,

Submit your manuscript here: http://www.dovepress.com/lung-cancer-targets-therapy-journal detection and screening; Cellular research and biomarkers; Identification of biotargets and agents with novel mechanisms of action; Optimal clinical use of existing anticancer agents, including combination therapies; Radiation and surgery; Palliative care; Patient adherence, quality of life, satisfaction; Health economic evaluations. 